\title{
Genotipificación de cagA y de la región intermedia de vacA en cepas de Helicobacter pylori aisladas de pacientes adultos colombianos y su asociación con enfermedades gástricas
}

\section{Genotyping of cagA and the intermediate region of vacA in strains of Helicobacter pylori isolated from Colombian adult patients and associations with gastric diseases}

\author{
M. Camila Melo-Narváez,, Diana F. Rojas-Rengifo, ${ }^{1}$ Luisa F. Jiménez-Soto, ${ }^{2}$ María del Pilar Delgado, ${ }^{1}$ Belén Mendoza de Molano, ${ }^{3}$ \\ José Fernando Vera-Chamorro, ${ }^{3}$ Carlos Jaramillo. ${ }^{1}$
}

\author{
Laboratorio de Diagnóstico Molecular y \\ Bioinformática (LDMB). Universidad de los Andes. \\ Bogotá D.C., Colombia. \\ 2 Max von Pettenkoffer Institute for Hygiene and \\ Clinical Microbiology. LMU München. Department of \\ Bacteriology. Múnich, Alemania. \\ 3 División de Gastroenterología y Endoscopía \\ Digestiva. Fundación de Santa Fe de Bogotá. Bogotá \\ D. C., Colombia \\ Correspondencia: María Camila Melo Narváez. Correo: \\ cami93melo@gmail.com (Bogotá D. C., Colombia). \\ Fecha recibido: $28-09-17$ \\ Fecha aceptado: 13-04-18
}

\section{Resumen}

Objetivo: este estudio caracteriza la diversidad de los genes de virulencia cagA (gen asociado con la citotoxina A) y vacA (citotoxina vacuolizante) en pacientes colombianos para determinar posibles asociaciones entre estos 2 genes y la severidad de los hallazgos endoscópicos teniendo en cuenta todos los genotipos reportados para el gen vacA (s, m e i). Materiales y métodos: Helicobacter pylori fue detectado por cultivo y por métodos moleculares en biopsias de 62 pacientes. Los genotipos de cagA y vacA $(\mathrm{m} / \mathrm{i} / \mathrm{s})$ se determinaron por reacción en cadena de la polimerasa (PCR) y secuenciación. Resultados: se aislaron 124 cepas de 62 pacientes; de estas, el 48,5\% $(\mathrm{n}=48)$ fueron vacA s2/m2/i2-cagA (-) presente en su mayoría en pacientes con gastritis folicular; mientras el $32,3 \%$ ( $n=32$ ) fueron vacA s $1 / m 1 / 11$-cagA (+) presentes mayormente en pacientes con gastritis folicular, gastritis crónica y posible metaplasia. Se encontró una asociación significativa entre la presencia de cagA y el genotipo $\operatorname{vac} A$ s1/m1/i1 y la ausencia de cagA y el genotipo vacA s2/m2/i2 ( $<<0,001)$. No se encontró una asociación significativa entre la severidad de los hallazgos endoscópicos y el estatus cagA-vacA de las cepas. Conclusión: se encontró una baja prevalencia de cepas cagA (+), el estatus cagA-vacA no es un predictor de riesgo en la población estudiada y la presencia de infecciones heterogéneas sin tropismo sugieren la necesidad de tomar biopsias tanto del cuerpo como del antro del estómago en la práctica clínica rutinaria.

\section{Palabras clave}

Subtipos de vacA, cagA, Helicobacter pylori, Colombia.

\begin{abstract}
Objective: This study characterizes the diversity of cagA and vacA virulence genes in Colombian patients to determine possible associations between them and the severity of endoscopic findings. It considers all four genotypes reported for the vacA gene ( $s, m$ and i). Materials and methods: Helicobacter pylori was detected in biopsies of 62 patients through culturing and by molecular methods. Genotypes of cagA and vacA $(\mathrm{m} / \mathrm{i} / \mathrm{s})$ were determined by PCR and sequencing. Results: One hundred twenty four strains from 62 patients were isolated. Of these, $48.5 \%(n=48)$ were vacA s2/m2/i2 - cagA $(-)$ which were mostly found in patients with follicular gastritis; $32.3 \%(n=32)$ were vacA s1/m1/i1-cagA $(+)$ which were mostly found in patients with follicular gastritis, chronic gastritis and possible metaplasia. Significant associations were found between the presence of cagA and the vacA s1/m1/i1 genotype and the absence of cag $A$ and the vacA s2/m2/i2 genotype $(p<0.001)$. No significant association was found between the severity of endoscopic findings and the cagAvacA status of the strains. Conclusion: We found a low prevalence of $\operatorname{cag} A(+)$ strains, the $\operatorname{cag} A$-vacA status is not a predictor of risk in this population. Moreover, the presence of heterogenous infections without tropism suggests a need for biopsies from both the corpus and the antrum of the stomach in routine clinical practice.
\end{abstract}

\section{Keywords}

vacA subtypes, cagA, Helicobacter pylori, Colombia. 


\section{INTRODUCCIÓN}

Helicobacter pylori es una bacteria gram-negativa, de forma espiral y microaerofílica que infecta a más del $50 \%$ de la población mundial (1). La infección se ha asociado con enfermedades como gastritis, úlcera péptica, linfoma asociado con mucosa (MALT) y adenocarcinoma gástrico (2). Los factores de virulencia más importantes de H. pylori son el gen asociado con la citotoxina A (CagA) y la citotoxina vacuolizante A (VacA) (3). Según la presencia del gen cagA, las cepas se clasifican en positivas o tipo I, asociadas con una mayor virulencia debido al daño gástrico visible inducido; y negativas o de tipo II, asociadas con menor virulencia, actuando más como bacterias comensales que como patógenas (4).

La proteína VacA causa una serie de alteraciones en las células gástricas humanas, incluidas la formación de vacuolas citoplasmáticas $(5,6)$, permeabilización de la membrana plasmática (6), secreción mitocondrial del citocromo c (7), fragmentación mitocondrial (8), activación de quinasas activadas por mitógenos (9) e inducción de autofagia (6); y ha sido clasificada como proteína inmunomoduladora $(6,10)$. El gen vacA tiene 3 regiones polimórficas llamadas región señal $(s)$, región intermedia $(i)$ y región media $(m)$, involucradas en el desarrollo de la enfermedad (2, 3, 6, 11-13).

La región $s$ está implicada en la eficiencia de la formación de canales y la región media $(m)$ afecta el tropismo hacia las células huésped (3). La región intermedia ( $i$ ) se describió recientemente y se ha observado que la actividad vacuolizante de la variante VacA il es más fuerte que la del subtipo VacA i2 $(3,6)$. Algunos análisis de la secuencia de nucleótidos y aminoácidos de este gen han revelado polimorfismos agrupados en los clústeres A, B y C, de los cuales los aminoácidos en los clústeres $\mathrm{B}$ y $\mathrm{C}$ son los responsables de la actividad vacuolizante de la proteína (11).

Para clasificar una cepa como i1, i2 o i3 se compara la secuencia de aminoácidos con las secuencias de las cepas de referencia (60190 para il y Tx30a para i2). La variante vacA i3 incluye cepas, cuyo clúster B es similar al $i 1$ y el clúster $\mathrm{C}$ al $i 2$ (11). Se han descrito diferentes combinaciones de las 3 regiones: las cepas vacA $s 1 / m 1$ con actividad vacuolizante y asociadas con un mayor riesgo de atrofia gástrica y adenocarcinoma en comparación con las cepas vacA $s 2 / m 2$ menos virulentas $(11,12)$. Asimismo, se ha descrito que las cepas vacA il están asociadas con adenocarcinoma gástrico $(2,11,12)$.

Recientemente, estudios epidemiológicos han sugerido que la interacción entre los factores de virulencia cagA y vacA (6), la susceptibilidad del huésped como diferencias entre hombres y mujeres (14-16) y factores ambientales modulan el desarrollo de la enfermedad $(4,12)$.

Se han publicado varios estudios de genotipificación de aislamientos de $\mathrm{H}$. pylori de diferentes poblaciones colom- bianas. Teniendo en cuenta que la región intermedia puede cambiar el comportamiento de la toxina, este estudio complementa los datos sobre la genotipificación de estas 2 toxinas, incluye la caracterización de esta región de vacA en aislamientos en una población andina colombiana con enfermedades gástricas de diferente severidad y evalúa las correlaciones entre el genotipo y el desarrollo de la enfermedad gástrica.

\section{MATERIALES Y MÉTODOS}

\section{Pacientes y población de estudio}

Se incluyeron 62 pacientes mayores de 18 años infectados por H. pylori en Bogotá (Colombia) con sintomatologías gástricas e indicación de endoscopia. Se excluyeron pacientes que hubieran ingerido inhibidores de la bomba de protones (IBP) en los últimos 15 días, antiácidos 12 horas antes del procedimiento o antibióticos en el mes anterior; que padecieran enfermedades cardiovasculares y respiratorias; pacientes con cáncer, sometidos a radioterapia o quimioterapia en los 6 meses previos al procedimiento; $y$ pacientes con coagulopatías o amiloidosis.

\section{Cepas y purificación del ADN}

Las biopsias se maceraron y se sembró una dilución $10^{-1}$ en agar GC (Oxoid, Alemania) suplementado con colesterol (1X, Gibco) y DENT (Oxoid, Alemania) (17). Se incubaron a $37^{\circ} \mathrm{C}$ en una atmósfera controlada con $10 \%$ de dióxido de carbono $\left(\mathrm{CO}_{2}\right)$ durante 4 a 10 días. Para la extracción de ADN se usó el kit Quick-gDNA Miniprep (Zymo Research, California, Estados Unidos) según las instrucciones del fabricante. Las cepas se seleccionaron por cultivo y se confirmaron como $H$. pylori mediante reacción en cadena de la polimerasa (PCR) del gen $23 \mathrm{~S}$ con los cebadores HPY-S y HPY-A reportados previamente (18).

\section{Detección de cagA}

Se realizó una PCR con los cebadores diseñados en este estudio y el protocolo previamente reportado (19) modificado y descrito en la Tabla 1. La PCR tuvo un volumen final de $25 \mu \mathrm{L}$, con GoTaq Green Master Mix 1X (Promega, Wisconsin, Estados Unidos) y 0,25 mM de cada cebador. La visualización se realizó en geles de agarosa al 1,5\%.

\section{Genotipificación de vacA}

Las 3 regiones polimórficas del gen vacA se caracterizaron por medio de PCR con un volumen final de $25 \mu \mathrm{L}$, que contenía GoTaq Green Master Mix 1X (Promega, Wisconsin, 
Tabla 1. Cebadores y protocolo usado para la amplificación de cagA y vacA

\begin{tabular}{|c|c|c|c|c|}
\hline Región & Primer & Secuencia & $\begin{array}{l}\text { Tamaño del } \\
\text { producto }(\mathrm{pb})\end{array}$ & Protocolo PCR \\
\hline \multirow[t]{2}{*}{ cagA } & DR1 & GATAACAGGCAAGCTTTTGAGG & \multirow[t]{2}{*}{179} & \multirow{2}{*}{$\begin{array}{l}1 \text { ciclo de } 94^{\circ} \mathrm{C} \text { por } 4 \text { min, } 32 \text { ciclos de: } 94^{\circ} \mathrm{C} \text { por } 30 \mathrm{~s}, 52^{\circ} \mathrm{C} \text { por } 30 \mathrm{~s} \mathrm{y} \\
72^{\circ} \mathrm{C} \text { por } 1 \text { min, y } 1 \text { ciclo de } 72{ }^{\circ} \mathrm{C} \text { durante } 10 \mathrm{~min}(19)\end{array}$} \\
\hline & DR2 & CTGCAAAAGATTGTTTGGCAGA & & \\
\hline \multirow{2}{*}{$\begin{array}{l}\operatorname{vac} A s \\
(19)\end{array}$} & Va1-F & ATGGAAATACAACAAACACAC & s1: 259 & \multirow{2}{*}{$\begin{array}{l}1 \text { ciclo de } 94^{\circ} \mathrm{C} \text { durante } 4 \text { min, } 30 \text { ciclos de } 94^{\circ} \mathrm{C} \text { durante } 30 \mathrm{~s}, 58^{\circ} \mathrm{C} \\
\text { durante } 30 \text { s y } 72^{\circ} \mathrm{C} \text { durante } 1 \mathrm{~min} \text {, y } 1 \text { ciclo de } 72^{\circ} \mathrm{C} \text { durante } 10 \text { min (19) }\end{array}$} \\
\hline & Va1-R & CTGCTTGAATGC GCCAAAC & s2: 289 & \\
\hline \multirow{2}{*}{$\begin{array}{l}\operatorname{vac} A m 1 \\
(19)\end{array}$} & Va3-F & GGTCAAAATGCGGTCATGG & 290 & \multirow{4}{*}{$\begin{array}{l}1 \text { ciclo de } 94^{\circ} \mathrm{C} \text { durante } 4 \text { min, } 30 \text { ciclos de } 94^{\circ} \mathrm{C} \text { durante } 30 \mathrm{~s}, 55^{\circ} \mathrm{C} \\
\text { durante } 30 \text { s y } 72^{\circ} \mathrm{C} \text { durante } 1 \text { min, y } 1 \text { ciclo de } 72^{\circ} \mathrm{C} \text { durante } 10 \text { min (19) }\end{array}$} \\
\hline & Va3-R & CCATTGGTACCT GTAGAAAC & & \\
\hline \multirow{2}{*}{$\begin{array}{l}\operatorname{vacA} m 2 \\
(19)\end{array}$} & Va4-F & GGAGCCCCAGGAAACATTG & \multirow[t]{2}{*}{352} & \\
\hline & Va4-R & CATAACTAGCGTCTTGCAC & & \\
\hline \multirow{2}{*}{$\begin{array}{l}\operatorname{vacAi1} \\
(2)\end{array}$} & VacF1 & GTTGGGATTGGGGGAATGCCG & \multirow[t]{2}{*}{426} & \multirow{4}{*}{$\begin{array}{l}1 \text { ciclo de } 95^{\circ} \mathrm{C} \text { durante } 4 \text { min, } 35 \text { ciclos de } 95^{\circ} \mathrm{C} \text { durante } 30 \mathrm{~s}, 55^{\circ} \mathrm{C} \\
\text { durante } 60 \mathrm{~s} \text { y } 72{ }^{\circ} \mathrm{C} \text { durante } 30 \mathrm{~s} \text {, y } 1 \text { ciclo de } 72^{\circ} \mathrm{C} \text { durante } 5 \text { min (2) }\end{array}$} \\
\hline & $\mathrm{C} 1 \mathrm{R}$ & TTAATTTAACGCTGT TTGAAG & & \\
\hline \multirow{2}{*}{$\begin{array}{l}\operatorname{vacA} i 2 \\
(2)\end{array}$} & VacF1 & GTTGGGATTGGGGGAATGCCG & \multirow[t]{2}{*}{432} & \\
\hline & $\mathrm{C} 2 \mathrm{R}$ & GATCAACGCTCTGAT TTGA & & \\
\hline
\end{tabular}

pb: pares de bases.

Estados Unidos) y $0,25 \mathrm{mM}$ de los cebadores correspondientes para cada región, según los protocolos previamente reportados y modificados en este estudio (Tabla 1).

Para la visualización de los productos se usaron geles de agarosa al $2 \%$ (regiones $m, i$ ) y poliacrilamida al $10 \%$ (29: 1) (región $s$ ). Los geles de policacrilamida se tiñeron con una solución de GelRed 3X ( $15 \mu \mathrm{L}$ de Gelred $10000 \mathrm{X}$ y 5 $\mathrm{mL}$ de cloruro de sodio $[\mathrm{NaCl}] \mathrm{MM}$ ) durante 1 hora bajo agitación constante a temperatura ambiente.

Se secuenciaron las muestras que tuvieron un resultado positivo para ambos subtipos (vacA i1 e i2) (Macrogen, Seúl, Corea). El análisis de secuencias se realizó con el software CLC Genomics Workbench 10 y se comparó la secuencia de aminoácidos con las secuencias de referencia 60190 (GenBank No. U05676) y Tx30a (GenBank No. U29401). Se definieron las cepas como $i 3$ cuando el clúster $\mathrm{B}$ fue similar a la cepa de referencia $i 1$ y el clúster $C$ fue similar a la cepa de referencia i2 (11).

\section{Análisis estadísticos}

Se utilizó una prueba de Fisher para determinar posibles asociaciones entre el estado cagA y el genotipo $v a c A$ de las cepas, y se utilizó un modelo lineal generalizado para determinar con cuál de las variables había una asociación significativa. El mismo análisis estadístico se realizó entre las regiones polimórficas del gen $v a c A$ y el estatus $c a g A ;$ y entre el estatus cagA, los subtipos del gen $v a c A$ y la gravedad de los hallazgos endoscópicos. Se utilizó un chi cuadrado $\left(\chi^{2}\right)$ para determinar la posible asociación entre el sexo del paciente y el estatus cagA, y el sexo del paciente y la gravedad de los hallazgos endoscópicos. Los hallazgos endoscó- picos se separaron en 2 grupos: enfermedad gástrica leve y enfermedad gástrica severa (Tabla 2). Para el análisis se utilizó el software R (20) y se consideró significativo un valor $\mathrm{p}<0,05$.

Tabla 2. Clasificación de los hallazgos endoscópicos*

\begin{tabular}{llc}
\hline Severidad & \multicolumn{1}{c}{ Hallazgos endoscópicos (\%) } & $\begin{array}{c}\text { Edad promedio } \\
\text { (años) }\end{array}$ \\
\hline Leve & Sospecha de gastroparesias $(1,61)$ & 89 \\
& Gastritis crónica y aguda $(12,9)$ & $53,7 \pm 9,66$ \\
& Gastritis crónica $(16,1)$ & $58,8 \pm 17,3$ \\
& Gastritis folicular $(27,4)$ & $39,8 \pm 12,4$ \\
Severo & Gastritis petequial $(4,84)$ & $48,6 \pm 24,1$ \\
& Gastritis erosiva $(17,7)$ & $52,5 \pm 14,6$ \\
& Gastritis atrófica $(6,45)$ & $58,5 \pm 19,3$ \\
& Úlcera gástrica $(1,61)$ & 58 \\
& Sospecha de metaplasia (11,2) & $45,1 \pm 13,1$ \\
\hline
\end{tabular}

*Se muestra la prevalencia de los diferentes hallazgos endoscópicos y cómo estos fueron clasificados en 2 grupos: leve o severo, según el criterio médico.

\section{RESULTADOS}

\section{Pacientes y aislamiento de $\boldsymbol{H}$. pylori}

Se analizaron 124 cepas identificadas como $H$. pylori provenientes de 62 pacientes con una edad promedio de 50,2 $\pm 16,2$ años con diferentes síntomas (Tabla 3 ) y cuya relación hombre-mujer fue de $1,48 / 1$. 
Tabla 3. Distribución de los síntomas gástricos reportados por los pacientes* $^{*}$

\begin{tabular}{lc}
\hline \multicolumn{1}{c}{ Síntoma } & Cantidad de pacientes (\%) \\
\hline Dolor epigástrico & $37(59,7)$ \\
Regurgitación & $32(51,6)$ \\
Dolor abdominal & $31(50,0)$ \\
Dolor esternal & $8(12,9)$ \\
Disfagia & $6(9,68)$ \\
Llenura postprandial & $10(16,1)$ \\
Pérdida de apetito & $1(1,61)$ \\
Pérdida de peso & $9(14,5)$ \\
Pirosis & $28(45,2)$ \\
Reflujo & $32(51,6)$ \\
\hline
\end{tabular}

*Los pacientes reportaron diferentes sintomatologías gástricas antes de la endoscopia digestiva.

\section{Genotipificación de cagA y vacA}

La presencia del gen cagA se pudo establecer mediante PCR con los cebadores correspondientes y $52(48,1 \%)$ de las 124 cepas analizadas fueron positivas para cagA. Sin embargo, no se encontró una asociación significativa entre la severidad de los hallazgos endoscópicos y la presencia de cagA $\left(\chi^{2}, \mathrm{p}>0,05\right)$ (Tabla 4). Tampoco se encontró una asociación significativa entre el sexo de los pacientes y la presencia de cagA $\left(\chi^{2}, \mathrm{p}>0,05\right)$ ni entre la gravedad de los hallazgos endoscópicos y el sexo (prueba exacta de Fisher, $\mathrm{p}>0,05)$ o la presencia de cagA $\left(\chi^{2}, \mathrm{p}>0,05\right)$ (Tabla 4).

Tabla 4. Asociación entre la severidad de los hallazgos endoscópicos, el estatus cagA y las regiones polimórficas del gen $v a c A^{*}$

\begin{tabular}{cccc}
\hline \multicolumn{2}{c}{$\begin{array}{c}\text { Severidad del hallazgo } \\
\text { endoscópico }\end{array}$} & Leve $\mathrm{n}(\%)$ & Severo $\mathrm{n}(\%)$ \\
\hline cagA & Positivo & $11(27,5)$ & $6(15,0)$ \\
\multirow{4}{*}{$\operatorname{vacA}$} & Negativo & $15(37,5)$ & $8(20,0)$ \\
& $s 1$ & $10(25,0)$ & $8(20,0)$ \\
& $s 2$ & $16(40,0)$ & $6(15,0)$ \\
$m 1$ & $9(22,5)$ & $6(15,0)$ \\
& $m 2$ & $17(42,5)$ & $8(20,0)$ \\
& $i 1$ & $10(25,0)$ & $6(15,0)$ \\
& $i 2$ & $16(40,0)$ & $8(20,0)$ \\
& $s 1 / m 1 / i 1$ & $8(20,0)$ & $6(15,0)$ \\
$s 1 / m 2 / i 1$ & $1(2,50)$ & $0(0,0)$ \\
$s 1 / m 2 / i 2$ & $1(2,50)$ & $2(5,0)$ \\
$s 2 / m 1 / i 1$ & $1(2,50)$ & $0(0,0)$ \\
$s 2 / m 2 / i 2$ & $15(37,5)$ & $6(15,0)$ \\
\hline
\end{tabular}

*No se encontró una relación significativa entre la severidad de los hallazgos endoscópicos y estatus cagA-vacA. Se excluyeron a los pacientes con genotipos diferentes entre antro y cuerpo. Prueba exacta de Fisher y modelo lineal generalizado binomial $(p>0,05)$.
En el caso de $v a c A$, se descartaron 16 cepas $(\mathrm{n}=8$ pacientes) en las que no fue posible determinar el genotipo para la región $s: \mathrm{n}=3(37,5 \%)$, la región $m: \mathrm{n}=3(37,5 \%)$, las regiones $s$ e $i: \mathrm{n}=1(12,5 \%)$ o ninguna de las regiones: $\mathrm{n}=1$ $(12,5 \%)$. De las 108 cepas restantes, 50 cepas fueron $s 1$ y 58 $s 2,41$ cepas fueron $m 1$ y $67 m 2$, y 34 cepas fueron $i 1$ y $55 i 2$. Se secuenciaron 19 muestras $(17,6 \%)$ que mostraron una amplificación para ambos subtipos vacA il e i2 (GenBank No. MF457450 a MF457477). De estas, 4 fueron clasificadas como i1, 3 como i2, 3 como i3 y 9 como ili2; estas últimas no se tuvieron en cuenta para los análisis posteriores. No se encontró una asociación significativa entre los subtipos vacA o el genotipo completo de $v a c A$ y la gravedad de los hallazgos endoscópicos ( $\left.\chi^{2}, \mathrm{p}>0,05\right)$ (Tabla 4).

Para evaluar la relación entre los 2 genes se descartaron 28 cepas $(22,6 \%)$ debido a que tenían un genotipo diferente para cagA y vacA entre antro y cuerpo. No se encontró un tropismo o correlación significativa entre el genotipo y la ubicación dentro del estómago de estas cepas. Sin embargo, se encontró una asociación significativa entre el estado cagA y los subtipos del gen $v a c A$ por separado (prueba exacta de Fisher, $\mathrm{p}<0,05$ ) (Figura 1) y combinados así: $s 1 / \mathrm{ml}$ / $i 1$-cagA positivo (32,3\%), s1/m2/i1-cagA positivo (2,02\%) y $s 1 / m 2 / i 2(7,07 \%)$; de los cuales el $71,4 \%$ fue cagA positivo, $s 1 / \mathrm{m} 1 /$ i3-cagA positivo (2,02\%), s1/m2/i3-cagA positivo (1,01\%), s2/m1/i1-cagA positivo $(2,02 \%)$ y $s 2 /$ $m 2 / i 2$-cagA negativo (48,5\%) (prueba exacta de Fisher, $\mathrm{p}<0,001$; modelo lineal generalizado binomial $\mathrm{p}<0,05$ ). Además, todas las cepas vacA i3 fueron cag $A$ positivas.

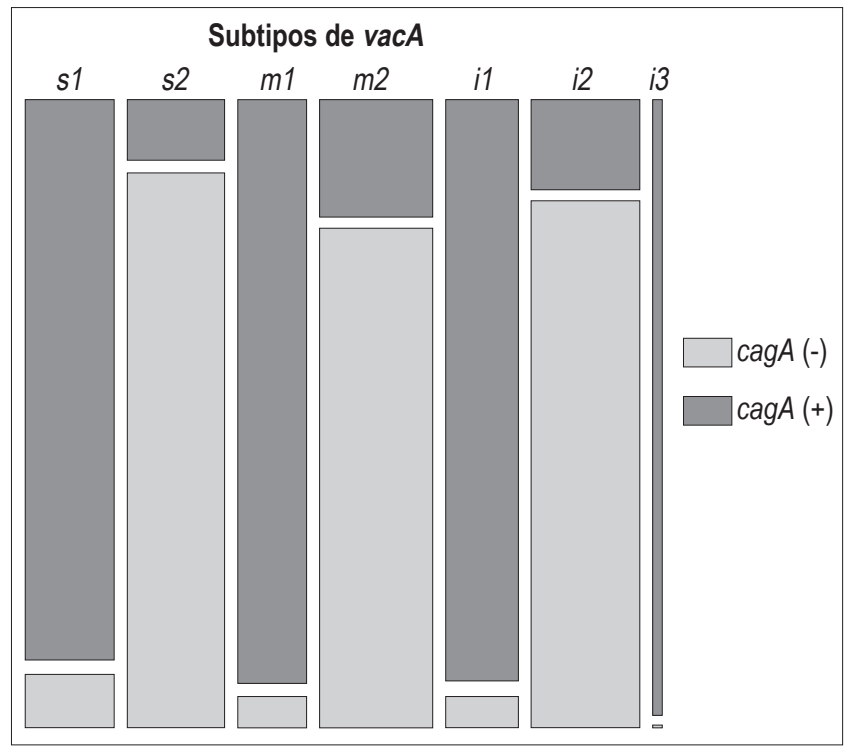

Figura 1. Asociación entre el estado cagA y los subtipos vacA. Existe una asociación significativa entre el estado cagA y los subtipos de vacA. Se excluyeron pacientes con estatus cagA o genotipos de $v a c A$ diferentes entre el antro y el cuerpo. Prueba exacta de Fisher y modelo lineal generalizado binomial $(\mathrm{p}<0,001)$. 


\section{DISCUSIÓN}

Este estudio caracteriza la diversidad de los genes de virulencia cagA y $v a c A$ en pacientes colombianos para determinar las posibles asociaciones entre estos 2 genes y la severidad de los hallazgos endoscópicos considerando todos los genotipos reportados para el gen vacA $(s, m$ e $i)$. Durante la infección por $H$. pylori, su variabilidad genética junto con factores del hospedero y del medio ambiente juegan un papel fundamental en el desarrollo de las patologías (3, 4, $6,12,21-23)$. De acuerdo con esto, se ha reportado que los pacientes infectados con cepas vacA $s 1 / \mathrm{ml} / \mathrm{il}$ - cagA (+) se encuentran en mayor riesgo de desarrollar carcinomas gástricos que aquellos pacientes infectados con cepas menos virulentas vacA s2/m2/i2-cagA (-) $(2,4,11-13,19,24)$.

En la población colombiana estudiada, se encontró una prevalencia del $48,1 \%$ de los pacientes infectados con cepas cagA positivo, inferior a lo previamente reportado en estudios realizados en Bogotá, Colombia, que alcanzaban una prevalencia del 73\% (24). Sin embargo, es similar a la reportada en pacientes provenientes de Tolima, Colombia, con un $43 \%$ de cepas cagA positivo (25). La prevalencia de este grupo estudiado difiere también de lo encontrado en otras regiones del mundo como Senegal (73,3\%) (26), Medio Oriente (100\%), Estados Unidos (80\%), Europa Occidental y América Latina (60\%-70\%) (11). Esto probablemente se debe a que la población seleccionada era de clase socioeconómica media y alta, pues se ha visto que la prevalencia de la infección es mayor en los niveles socioeconómicos bajos, en los que existen pobres condiciones sanitarias $(27,28)$.

Este estudio muestra también que el 22,6\% de los pacientes presentaba infecciones por cepas con un genotipo diferente para cagA y vacA entre las 2 locaciones del estómago estudiadas (antro y cuerpo) sin ningún tropismo. Es posible que estos pacientes hayan sido infectados en ocasiones diferentes por diferentes cepas de H. pylori (29), debido a que la prevalencia de infecciones múltiples parece ser mayor en lugares donde el riesgo de infección por H. pylori es alto, como en Colombia (30). La presencia de este tipo de infecciones reafirma la necesidad de toma de biopsias en cuerpo y antro de forma rutinaria.

La relación entre la presencia de cagA y del genotipo $v a c A$ $s 1$ ha sido descrita por diferentes autores $(31,32)$. En este estudio, el $68,1 \%$ de las cepas de cagA positivo fue vacA s1/ $m 1 / i 1$ y solo el $6,4 \%$ fue $v a c A s 2 / m 2 / i 2$. De este modo, el genotipo vacA $s 1 / \mathrm{ml} / \mathrm{il}$ está significativamente asociado con la presencia del gen cagA. En el caso de las cepas vacA $s 1 / m 2$, se ha observado que hay una mayor prevalencia de cepas tipo $i 1$ o $i 2$ dependiendo de la región geográfica (2, 26). Se encontró una baja prevalencia de la combinación vacA $s 2 / m 1$, lo que concuerda con lo descrito previamente, que sugiere que la baja prevalencia de este genotipo podría explicarse por una selección negativa de este subtipo por ser desfavorable para los microorganismos (26).

Además, se describe la región intermedia del gen vacA en las cepas circulantes en una población colombiana. Casi el total de cepas vacA $s 1 / m 1$ (94,4\%) fueron il y todas la cepas vacA $s 2 / m 2$ fueron $i 2$, en concordancia con lo reportado en la primera descripción de la región intermedia (2). La mayoría de las cepas analizadas fueron vacA i2 y se encontraron 3 pacientes infectados por cepas vacA i3 definidas como aquellas con el clúster B similar a il y el clúster $\mathrm{C}$ similar al tipo i2 (11). La variante $i 3$ podría reflejar un proceso de recombinación entre las cepas de tipo il e i2 $(6,11)$, pero la importancia clínica o patológica de esta variante sigue siendo desconocida y en nuestro estudio no se encontró ninguna asociación significativa con los hallazgos endoscópicos. Sin embargo, todas las cepas vacA i3 fueron cagA positivo. Sería necesaria una muestra más grande de pacientes infectados por cepas i3 para entender mejor la naturaleza de este genotipo y el desarrollo de la enfermedad en estos pacientes.

Contrario a la asociación significativa entre el genotipo vacA s1/m1/i1-cagA (+) y algunas enfermedades digestivas severas como la gastritis atrófica, úlceras y adenocarcinoma gástrico observada en otros estudios (33); no se encontró ninguna asociación significativa entre el estatus cagA-vacA de las cepas y la severidad de los hallazgos endoscópicos, a pesar de que la mayor parte de las cepas aisladas fueron vacA $s 2 / m 2 / i 2$ - cagA (-) provenientes de pacientes que en su mayoría presentaban gastritis folicular (Figura 2). Lo anterior pudo ser el resultado del tamaño reducido de la muestra para cada una de las categorías de la severidad de los hallazgos endoscópicos (leve o severo) y de la alta prevalencia de cepas tipo II en los pacientes. Por este motivo, el genotipo de $v a c A$ no puede utilizarse para identificar pacientes de alto riesgo en la población estudiada.

En conclusión, este estudio brinda información nueva sobre la prevalencia de los factores de virulencia cagA y vacA, y su asociación con los hallazgos endoscópicos, debido a que estudios realizados en la costa occidental y en el sur de los Andes difieren de lo encontrado en la presente investigación $(34,35)$. Lo anterior recalca la necesidad de incrementar los estudios comparativos entre diferentes regiones y poblaciones colombianas, porque los datos publicados en Colombia muestran una alta variabilidad en la presencia de marcadores genéticos de patogenicidad y su correlación con la severidad de patologías gástricas.

Por su parte, algunos estudios recientes indican una evolución independiente de las cepas de $H$. pylori en la población colombiana originándose en la línea europea (HpEurope) (36). Es probable que los típicos mecanismos moleculares asociados con enfermedades gástricas severas 


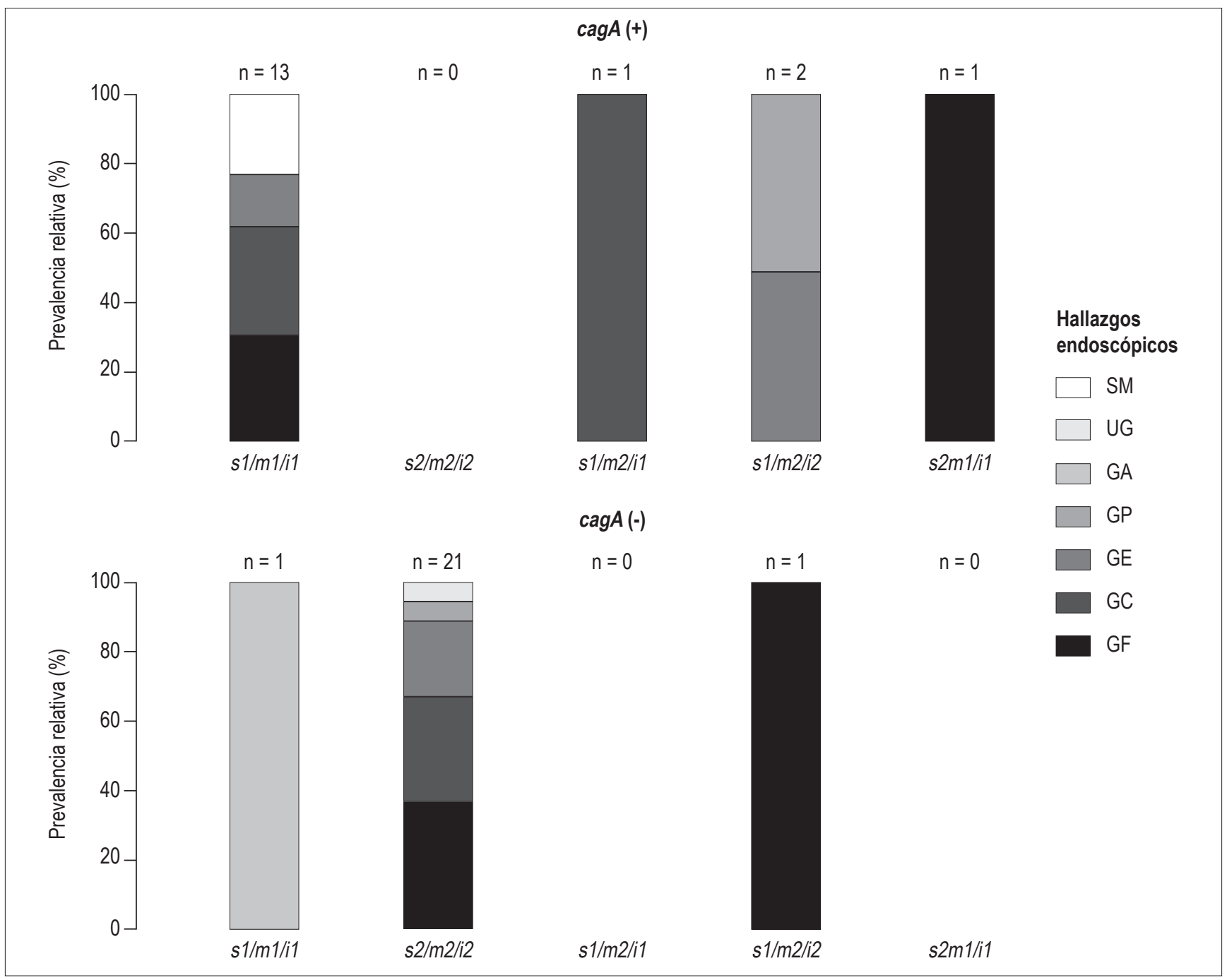

Figura 2. Prevalencia relativa de los hallazgos endoscópicos según el genotipo de las cepas de H. pylori aisladas en estos pacientes. El 52,5\% de pacientes estaba infectado por cepas de $H$. pylori vacA $s 2 / m 2 / i 2$-cagA negativo relacionadas en su mayoría con gastritis folicular. Se excluyeron pacientes con estatus cagA o genotipos de vacA diferentes entre el antro y el cuerpo. Sospecha de metaplasia (SM), úlcera gástrica (UG), gastritis atrófica (GA), gastritis petequial (GP), gastritis erosiva (GE), gastritis crónica (GC) y gastritis folicular (GF).

asociadas con la presencia de esta bacteria se comporten diferente en la población colombiana gracias a las diferentes ancestrías presentes (37). Si se considera la relación bacteria-huésped, es posible que la falta de correlación entre cagA y vacA, y la patología de la infección en comparación con otros estudios se pueda investigar a fondo si se considera en futuros estudios la distribución ancestral presente en las regiones de Colombia.

\section{Agradecimientos}

Los autores quieren agradecerle a Sebastián Posada, MD, Laura Camila Zuluaga, MD, Daniela Campaz, MD y
Manuel Arrieta, MD por su ayuda en la recolección de muestras y el manejo de la base de datos. Queremos agradecerle a Verónica Arévalo Jaimes por su ayuda en el procesamiento molecular e identificación de las cepas por medio de PCR de la región 23S. Les agradecemos al Profesor Dr. Adolfo Amézquita y a Cindy Ulloa por su apoyo en los análisis estadísticos.

\section{Financiación}

Este estudio fue apoyado por la Facultad de Ciencias de la Universidad de los Andes bajo el proyecto titulado "Los factores de virulencia cagA y vacA de cepas de $H$. pylori y su 
relación con la patología en pacientes adultos colombianos sintomáticos", código: P16.160322.001/01-BIO01; decidido durante una sesión del Comité I\&P el 11/12/2015 y la beca de investigación del DFG (JI 221/1-1) para LFJ-S.

\section{REFERENCIAS}

1. Peleteiro B, Bastos A, Ferro A, et al. Prevalence of Helicobacter pylori infection worldwide: a systematic review of studies with national coverage. Dig Dis Sci. 2014;59(8):1698-709. doi: 10.1007/s10620-014-3063-0.

2. Rhead JL, Letley DP, Mohammadi M, et al. A new Helicobacter pylori vacuolating cytotoxin determinant, the intermediate region, is associated with gastric cancer. Gastroenterology. 2007;133(3):926-36. doi: 10.1053/j. gastro.2007.06.056.

3. Jones KR, Jang S, Chang JY, et al. Polymorphisms in the intermediate region of VacA impact Helicobacter pylori-induced disease development. J Clin Microbiol. 2011;49(1):101-10. doi: 10.1128/JCM.01782-10.

4. García CA, Barra TR, Delgado SC, et al. Genotipificación de aislados clínicos de Helicobacter pylori en base a genes asociados a virulencia cagA, vacA y babA2: primer aislamiento de una cepa babA2 positiva en pacientes chilenos. Rev Médica Chile. 2006;134(8):981-8. doi: 10.4067/ S0034-98872006000800006.

5. Cover TL, Blaser MJ. Purification and characterization of the vacuolating toxin from Helicobacter pylori. J Biol Chem. 1992;267(15):10570-5.

6. Kim I-J, Blanke SR. Remodeling the host environment: modulation of the gastric epithelium by the Helicobacter pylori vacuolating toxin (VacA). Front Cell Infect Microbiol. 2012;2:37. doi: 10.3389/fcimb.2012.00037.

7. Foo JH, Culvenor JG, Ferrero RL, et al. Both the p33 and p55 Subunits of the Helicobacter pylori VacA Toxin Are Targeted to Mammalian Mitochondria. J Mol Biol. 2010;401(5):792-8. doi: 10.1016/j.jmb.2010.06.065.

8. Jain P, Luo Z-Q, Blanke SR. Helicobacter pylori vacuolating cytotoxin A (VacA) engages the mitochondrial fission machinery to induce host cell death. Proc Natl Acad Sci U S A. 2011;108(38):16032-7. doi: 10.1073/pnas.1105175108.

9. Nakayama M, Kimura M, Wada A, et al. Helicobacter pylori VacA Activates the p38/Activating Transcription Factor 2-mediated Signal Pathway in AZ-521 Cells. J Biol Chem. 2004;279(8):7024-8. doi: 10.1074/jbc.M308898200.

10. Boncristiano M, Paccani SR, Barone S, et al. The Helicobacter pylori vacuolating toxin inhibits $\mathrm{T}$ cell activation by two independent mechanisms. J Exp Med. 2003;198(12):188797. doi: 10.1084 /jem.20030621.

11. Chung C, Olivares A, Torres E, et al. Diversity of vacA intermediate region among Helicobacter pylori strains from several regions of the world. J Clin Microbiol. 2010;48(3):6906. doi: 10.1128/JCM.01815-09.

12. Ferreira RM, Machado JC, Letley D, et al. A novel method for genotyping the Helicobacter pylori vacA Intermediate region directly in gastric biopsy specimens. J Clin Microbiol. 2012;50(12):3983-9. doi: 10.1128/JCM.02087-12.

13. González-Rivera C, Algood HMS, Radin JN, et al. The intermediate region of Helicobacter pylori vacA is a determinant of toxin potency in a jurkat $t$ cell assay. Infect Immun. 2012;80(8):2578-88. doi: 10.1128/IAI.00052-12.

14. Agah S, Khedmat H, Ghamar-Chehred ME, et al. Female gender and Helicobacter pylori infection, the most important predisposition factors in a cohort of gastric cancer: a longitudinal study. Casp J Intern Med. 2016;7(2):136-41.

15. Naja F, Kreiger N, Sullivan T. Helicobacter pylori infection in Ontario: Prevalence and risk factors. Can J Gastroenterol. 2007;21(8):501-6. doi: 10.1155/2007/462804.

16. Zhu Y, Zhou X, Wu J, et al. Risk factors and prevalence of Helicobacter pylori infection in persistent high incidence area of gastric carcinoma in Yangzhong City. Gastroenterol Res Pract. 2014;2014:e481365.

17. Jiménez-Soto LF, Rohrer S, Jain U, et al. Effects of cholesterol on Helicobacter pylori growth and virulence properties in vitro. Helicobacter. 2012;17(2):133-9. doi: 10.1111/j.1523-5378.2011.00926.x.

18. Ménard A, Santos A, Mégraud F, et al. PCR-restriction fragment length polymorphism can also detect point mutation $\mathrm{A} 2142 \mathrm{C}$ in the $23 \mathrm{~S}$ rRNA gene, associated with Helicobacter pylori resistance to clarithromycin. Antimicrob Agents Chemother. 2002;46(4):1156-7. doi: 10.1128/ AAC.46.4.1156-1157.2002.

19. Secka O, Antonio M, Berg DE, et al. Mixed infection with cagA positive and cagA negative strains of Helicobacter pylori lowers disease burden in The Gambia. PLoS ONE. 2011;6(11):e27954. doi: 10.1371/journal.pone.0027954.

20. R Development Core Team. R: A language and environment for statistical computing. R Foundation for Statistical Computing [internet] 2008 [acceso el 24 de noviembre de 2016]. Disponible en: http://www.R-project.org.

21. Argent RH, Thomas RJ, Letley DP, et al. Functional association between the Helicobacter pylori virulence factors vacA and cagA. J Med Microbiol. 2008;57(2):145-50. doi: 10.1099/jmm.0.47465-0.

22. Jang S, Jones $\mathrm{KR}$, Olsen $\mathrm{CH}$, et al. Epidemiological link between gastric disease and polymorphisms in vacA and cagA. J Clin Microbiol. 2010;48(2):559-67. doi: 10.1128/ JCM.01501-09.

23. Yamazaki S, Yamakawa A, Okuda T, et al. Distinct Diversity of vacA, cagA, and cagE Genes of Helicobacter pylori Associated with Peptic Ulcer in Japan.J Clin Microbiol.2005;43(8):390616. doi: 10.1128/JCM.43.8.3906-3916.2005.

24. Quiroga AJ, Cittely DM, Bravo MM. Frecuencia de los genotipos babA2, oipA y cagE de Helicobacter pylori en pacientes colombianos con enfermedades gastroduodenales. Biomédica. 2005;25:325-34. doi: 10.7705/biomedica. v25i3.1357.

25. Molina Delgado A, Jaramillo Henao C, Delgado Perafán M, et al. Detección y genotipificación de Helicobacter pylori sobre la base de los genes $\mathrm{ADNr} 16 \mathrm{~S}$ y el gen asociado a citotoxina ( $\operatorname{cag} \mathrm{A})$ y posible asociación con enfermedades 
gastrointestinales. Rev ista Cubana de Medicina Tropical. 2008;60:105-10.

26. Basso D, Zambon C-F, Letley DP, et al. Clinical relevance of Helicobacter pylori cagA and vacA gene polymorphisms. Gastroenterology. 2008;135(1):91-9. doi: 10.1053/j.gastro.2008.03.041.

27. Parente JML, da Silva BB, Palha-Dias MPS, et al. Helicobacter pylori infection in children of low and high socioeconomic status in northeastern Brazil. Am J Trop Med Hyg. 2006;75(3):509-12.

28. Ramírez Ramos A, Chinga Alayo E, Mendoza Requena D, et al. Variación de la prevalencia del H. pylori en el Perú período (1985-2002), en una población de nivel socioeconómico medio y alto. Rev Gastroenterol Perú. 2003;23(2):92-8.

29. Mendoza-Elizalde S, Cortés-Márquez AC, Giono-Cerezo $S$, et al. Analysis of the genotypic diversity of strains of Helicobacter pylori isolated from pediatric patients in Mexico. Infect Genet Evol. 2015;29:68-74. doi: 10.1016/j. meegid.2014.11.002.

30. Pinto-Ribeiro I, Ferreira RM, Batalha S, et al. Helicobacter pylori vacA genotypes in chronic gastritis and gastric carcinoma patients from Macau, China. Toxins (Basel). 2016;8(5). pii: E142. doi: 10.3390/toxins8050142.

31. Atherton JC, Cao P, Peek RM Jr, et al. Mosaicism in vacuolating cytotoxin alleles of Helicobacter pylori. Association of specific vacA types with cytotoxin production and peptic ulceration. J Biol Chem. 1995;270(30):1-7. doi: 10.1074/ jbc.270.30.17771.
32. Nagiyev T, Yula E, Abayli B, et al. Prevalence and genotypes of Helicobacter pylori in gastric biopsy specimens from patients with gastroduodenal pathologies in the Cukurova Region of Turkey. J Clin Microbiol. 2009;47(12):4150-3. doi: 10.1128/JCM.00605-09.

33. Breurec S, Michel R, Seck A, et al. Clinical relevance of cagA and vacA gene polymorphisms in Helicobacter pylori isolates from Senegalese patients. Clin Microbiol Infect. 2011;18(2):153-9. doi: 10.1111/j.1469-0691.2011.03524.x.

34. Bravo MM, Trujillo E, Quiroga A, et al. Genotipificación de Helicobacter pylori en individuos de dos regiones de Colombia con riesgo de cáncer gástrico opuesto. Rev Colomb Cancerol. 2013;17(4):178-9.

35. Nogueira C, Figueiredo C, Carneiro F, et al. Helicobacter pylori genotypes may determine gastric histopathology. Am J Pathol. 2001;158(2):647-54. doi: 10.1016/S00029440(10)64006-0.

36. Muñoz-Ramírez ZY, Mendez-Tenorio A, Kato I, et al. Whole genome sequence and phylogenetic analysis show Helicobacter pylori strains from Latin America have followed a unique evolution pathway. Front Cell Infect Microbiol. 2017;7:50. doi: 10.3389/fcimb.2017.00050.

37. Ossa H, Aquino J, Pereira R, et al. Outlining the ancestry landscape of Colombian admixed populations. PLoS One. 2016;11(10):e0164414. doi: 10.1371/journal. pone. 0164414 . 